\title{
La incorporación de la traducción colaborativa en la didáctica de la traducción ${ }^{1}$ \\ Introducing collaborative translation into translator training
}

\author{
MARÍA del MAR SÁNCHEZ RAMOS \\ mar.sanchezr@uah.es \\ Universidad de Alcalá de Henares
}

Fecha de recepción: 24 de octubre de 2018

Fecha de aceptación: 8 de febrero de 2019

Resumen: La evolución hacia formas de trabajo colaborativas representa un cambio de papeles en los procesos de traducción. Esta tendencia es un resultado directo de los cambios tecnológicos, sociales y culturales. El artículo describe cómo nuevas formas de traducción, como es la traducción colaborativa en línea, se ha incorporado como parte de la formación del traductor. Bajo un enfoque socioconstructivista y la metodología investigación-acción, el trabajo muestra datos cuantitativos y cualitativos derivados de la experiencia.

Palabras clave: traducción colaborativa en línea, tecnología y traducción, investigación-acción, didáctica de la traducción, estudios de traducción

Abstract: New collaborative tasks in translation represent a role reversal in translation processes as a consequence of technological, social and cultural changes. This article describes how new emerging trends in translation, such as online collaborative translation, have been introduced as part of the translator training syllabus. Based on a socioconstructivist framework and an action research methodology, the article presents the results of both quantitative and qualitative data.

Keywords: online collaborative translation, translation technology, action research, translation pedagogy, translation studies

\footnotetext{
${ }^{1}$ El trabajo desarrollado en este artículo se ha llevado a cabo como actividad investigadora dentro del grupo de innovación docente Herramientas Informáticas para la Traducción e Interpretación y su docencia-HITID, (UAH-GI14-71), Universidad de Alcalá (Madrid, España).
}

Hikma 18 (1) (2019), $261-281$ 


\section{INTRODUCCIÓN}

No hay duda de que estamos inmersos en la llamada era digital, donde cada día son más los ejemplos y avances que marcan el asentamiento y desarrollo de una revolución tecnológico-social; una era digital dirigida a la creación de prácticas lingüísticas completamente automatizadas e interconectadas. Dichas prácticas suponen una nueva percepción de los patrones tradicionales en el proceso de intercambio de comunicación, lo que, sin duda, incide en una reorganización de estrategias en el intercambio de la información y, en nuestro caso, un cambio en la práctica docente. Para ser más específicos, estos cambios nos llevan a planteamientos didácticos dentro del ámbito de la traducción y la tecnología, en donde el aprendizaje del componente tecnológico debe ser, y tomamos las palabras de Rico (2017), parte de un proyecto integral de aprendizaje.

Bajo este contexto, las prácticas traductoras se reorganizan en nuevos paradigmas de intercambio de comunicación: trabajo colaborativo, redes sociales, globalización de mercados, modelos de acceso a la información, flujo de trabajo, etc. Los consumidores de la información se transforman, a su vez, en agentes y actores de la misma, con lo que hay un cambio en las estrategias de producción de la comunicación y una diversificación de tareas. Un ejemplo de ello lo encontramos en el creciente interés despertado por la traducción realizada por voluntarios, con o sin formación en traducción (Cordingley y Manning, 2016; Jiménez Crespo, 2017). Desde que apareciera el artículo publicado por Howe (2006) en Wired Magazine, ha sido variada la terminología empleada para referirse a este modelo de traducción. Sirvan de ejemplo los términos en inglés social translación, community translation, crowdsourced translation o user-generated translation. A la vista de que este tipo de traducción tiene una influencia directa en la traducción desde diversos ámbitos, como puede ser el social y cultural (cuestiones éticas, relevancia de las lenguas minoritarias en la traducción colaborativa) hasta al propio ámbito institucional (implicaciones en la pedagogía de la traducción, impacto en la práctica profesional de la traducción), se hace necesaria una reflexión crítica desde nuestra disciplina.

El trabajo que seguidamente se describe expone el estudio realizado dentro programa de Grado en Lenguas Modernas y Traducción (Universidad de Alcalá), concretamente dentro de la asignatura llamada Herramientas informáticas aplicadas a la traducción durante el curso académico 2016-2017, cuyo principal objetivo es el de incorporar la traducción colaborativa en línea, como ejemplo de nuevas prácticas traductoras, dentro del sílabo de traducción y tecnología, además de conocer la percepción del estudiantado ante dichas prácticas. Tras esta introducción, el segundo apartado ofrece un panorama general de lo que la llegada de Internet y la tecnología ha supuesto

Hikma 18 (1) (2019), $261-281$ 
en el mundo profesional de la traducción. Seguidamente, nos detendremos en la llamada traducción colaborativa y las implicaciones que su práctica puede tener en un entorno de aprendizaje de la traducción. Posteriormente, se describe el estudio realizado (metodología, participantes y procedimiento) bajo una metodología de corte socioconstructivista y asentada en las bases de la investigación-acción. Finalmente, se concluye con los resultados obtenidos y la reflexión sobre la necesidad de incorporar nuevas modalidades de traducción, como es la traducción colaborativa en línea, dentro de la pedagogía de la traducción.

\section{TRADUCIR EN NUESTRO TIEMPO: ¿HACIA NUEVAS PRÁCTICAS TRADUCTORAS?}

Internet y la World Wide Web (WWW) son dos conceptos inseparables en nuestro día a día. Su influencia llega inexorablemente al mundo profesional y se convierten en dos conceptos indispensables en nuestro tiempo. El campo de la traducción no es ajeno a dicha influencia, y la llegada de la revolución digital marca nuevos retos y posibilidades. A su vez, Internet es el mayor repositorio de información, destacando por su rapidez y su fácil acceso, lo que facilita una de las tareas de comunicación, documentación y productividad (Jiménez Crespo, 2015).

Según O' Hagan (2013), la tecnología afecta a todo el ecosistema traductológico: influye en las herramientas y plataformas de traducción y en el proceso de traducción, con la ampliación de géneros textuales y modalidades o prácticas de traducción:

Technologies have significantly affected both the translators' microcosm, shaping their immediate local work environment, and also their macrocosm of global operating contexts in which new technologies are creating new content or products which require translation, as well as affording new ways of doing translation as illustrated by the current trend of crowdsourcing (Howe 2006, 2008) (O'Hagan, 2013: 503).

El llamado technological turn, acuñado por O' Hagan (2013), describe precisamente la influencia que la tecnología ejerce dentro de los estudios de traducción y, por ende, en la didáctica de la traducción. Los cambios acaecidos tanto en la forma de comunicarse como en el proceso de la traducción han llevado a nuevas modalidades y prácticas de traducción. De un lado, Internet ha hecho posible que la tipología textual amplíe sus fronteras hacia nuevos géneros textuales, como la localización web. De otro, entre las nuevas prácticas de traducción, como afirma Fernández Costales (2012), la denominada traducción colaborativa forma parte del panorama traductor de nuestra era. Además, el mismo autor es tajante, y no podemos estar más de 
acuerdo, cuando afirma que estas nuevas modalidades de traducción serán una práctica permanente: «we have to consider the possibility that community translation is here to stay» (2012: 22). Dentro de este panorama tecnológico, no solo florecen nuevos géneros textuales o nuevas modalidades de traducción, sino que hay un cambio en los propios actores implicados en el proceso de traducción. La evolución de la Web 1.0 hacia la Web 2.0, término acuñado por O'Reilly (2005) ha hecho posible que el usuario, que era pasivo en la era de la Web 1.0, se convierta en un usuario activo capaz de controlar y compartir información con otros usuarios de una forma que parecía inimaginable años atrás (Cronin, 2010). Se habla, de este modo, de la traducción generada por usuarios de Internet, cuya formación en traducción no es un requisito, que forman parte de una comunidad de traductores y que trabajan de forma voluntaria, en su mayoría en línea, sin búsqueda de remuneración económica para organizaciones sin ánimo de lucro.

El principal impacto de la tecnología puede verse en el desarrollo de nuevas herramientas $\mathrm{o}$ recursos documentales. Junto con las llamadas Herramientas de Traducción Asistida (TAO), los traductores disponen de multitud de herramientas informáticas, tales como bases de datos terminológicas, glosarios (monolingües, bilingües y multilingües), memorias de traducción compartidas, distintos tipos de corpus (monolingües, paralelos, comparables, etc.), diccionarios electrónicos, herramientas de traducción automática, y así un largo etcétera. Por otro lado, y como consecuencia de la globalización y el proceso de traducción como un intercambio de comunicación interlingüística e intercultural, la tecnología ha hecho posible el acceso de la información en distintos formatos. La traducción ha dejado de ser una disciplina centrada en la mediación lingüística para transformarse en una disciplina 'multimodal' (Fernández Costales, 2012). La traducción abarca nuevos ámbitos de actuación y, junto con la mediación lingüística, se centra en la adaptación de elementos no textuales, semióticos o culturales.

La incursión de la tecnología en la sociedad también ha supuesto cambios en lo que se refiere al acceso de la información. La traducción ha permitido que estemos inmersos en una sociedad multilingüe, donde todo (o casi todo) se traduce. Esta actividad se hace extensible a cualquier producto digital (programa informático, sitio web o videojuego). Definida como la adaptación lingüística y cultural de contenido digital a las necesidades de los distintos mercados (Schäler, 2011), la llamada localización tiene sus orígenes en la década de los 80 , cuando las primeras operaciones en la industria de la localización tuvieron lugar en los Estados Unidos. Compañías como Oracle o Microsoft fijaron otros países como clientes potenciales (Japón, Francia, Italia, Alemania o España), en parte debido al gran éxito de ventas de sus productos en dichos países. Con todo, el mundo académico no puede dar la 
espalda a esta actividad, y son muchos los programas formativos que existen y que ofrecen una formación especializada centrada en los procesos, herramientas y desarrollo de estrategias de localización (Folaron, 2006).

Traductores de todo el mundo forman comunidades que, inspiradas por diversos motivos, contribuyen a la generación de información multilingüe, desde material literario a programas informáticos. Este tipo de traducción voluntaria es especialmente relevante en cuestiones humanitarias y en situaciones de emergencia, donde miles de usuarios hacen posible que ciertas comunidades tengan acceso a información vital. Organizaciones como Permondo (España) o The Rossetta Foundation (Irlanda) o la Fundación Mozzila (Estados Unidos) tienen entre sus objetivos el de otorgar a los voluntarios un papel fundamental en la traducción y la localización. La traducción colaborativa representa, por tanto, un área que necesita ser explorada y que, como parte en los procesos de localización, implica el conocimiento de plataformas de trabajo colaborativas (i.e. Launchpad), sistemas de gestión de traducción en línea (Pootle, PoEditor) y formatos específicos (i.e. .po, portable object) para trabajar en procesos y proyectos de localización de programas de código abierto.

\section{TRADUCCIÓN COLABORATIVA Y DIDÁCTICA DE LA TRADUCCIÓN}

Debido, en gran parte, a la sociedad multicultural y multilingüe en la que vivimos, la industria de la traducción sigue su ascenso. La entrada de la traducción automática o los entornos colaborativos de traducción son buena muestra de la actual demanda de la traducción. Plataformas de gestión de proyectos que integran entornos colaborativos de traducción (Wordbee, PoEditor, SDL Cloud Translation Solutions) son un ejemplo (Drugan, 2013: 101). La rápida explosión de las tecnologías ha facilitado tanto el acceso a la información como la colaboración en tanto que trabajo conjunto en distintas tareas de forma desinteresada. No hay que remontarse muy lejos para afirmar que la colaboración en el mundo de la traducción profesional siempre ha existido, de una forma u otra (O'Brien, 2011). Tal y como afirma Gaspari (2015: 579), «usenet-based newsgroups were early online communities organized in hierarchical categories around topics of interests to their members, and designed to share textual messages via the nascent internet infraestructure». Este cambio denota una inversión papeles en el proceso social de la traducción. Como bien señala Cronin (2010: 4), «the consumer becomes an active producer or prosumer. It is no longer a question of the translator, for example, projecting a target-oriented model of translation on to an audience but the audience producing their own self-representation as a target audience». 
El fenómeno de la traducción colaborativa, sin entrar aun en la cuestión terminológica, es el resultado directo de los cambios sociales y culturales que nos rodean. Tawileh (2010: 2) habla de la traducción colaborativa como «an innovative approach that responds to the rapidly changing trends in content production and dissemination by transforming the translation process to become more open, inclusive and collaborative» y compañías como Twitter, Facebook, Google and TED Open Translation Project hacen uso de traductores voluntarios de todo el mundo (McDonough Dolmaya, 2012; Ray y Kelly, 2011).

Un tema que, sin duda, surge cuando se habla de traducción colaborativa es la cuestión terminológica. Proporcionar una definición concreta de esta modalidad de traducción es una tarea cuanto menos difícil y arriesgada, debido, en gran parte, a su carácter novedoso y su constante evolución. O' Hagan (2011) afirma que esta dificultad conceptual emana de su propia inestabilidad terminológica. Social translation (Tawileh, 2010: 2), wikitranslation (Cronin, 2010), volunteer translation o amateur translation (Pym, 2011), CT3 (community, crowdsourced and collaborative translation) (DePalma y Kelly, 2008) son algunos de los términos que se emplean para hacer referencia a esta actividad. Para el objeto de nuestro estudio, y con el fin de dotar a nuestro trabajo de una coherencia terminológica, nos decantamos por la distinción que algunos autores emplean, sin ser por ello reduccionistas (Jiménez Crespo, 2015; 2017; Fernández Costales, 2012, 2013): crowdsourcing translation y online collaborative translation. El neologismo crowdsourcing se atribuye a Howe (2006), quien lo define como «the act of taking a job traditionally performed by a designated agent (usually an employee) and outsourcing it to an undefined, generally large group of people in the form of an open call». Esta práctica suele relacionarse con distintas compañías e instituciones que intentan, de algún modo, reducir costes y cuyos principios legales y éticos no llegan a estar bien definidos. Dentro de la disciplina de los estudios de traducción, son varios los estudiosos que se han embarcado en la tarea de proporcionar una definición, más o menos exhaustiva, del término crowdsourcing. Tal es el caso de García (2010: 1), quien estrecha la definición primigenia de Howe (2006) y habla de este término como «roughly meaning the delegation to (unpaid) volunteers of tasks previously reserved for professionals». Otras definiciones, sin embargo, añaden más características a este negologismo. O' Hagan (2011) define crowdsourcing como el resultado de traductores voluntarios que conforman algún tipo de colaboración en forma de grupo de usuarios en Internet o comunidad online. Por otro lado, Declerqu (2014: 46) señala que esta nueva actividad se refiere a "the outsourcing of a task (or several tasks at the same time) to an undefined, generally large, group of people or community, mutually connected through an e-medium». En ambas definiciones, términos como 
colaboración, Internet y comunidad, estrechan y delimitan el campo de actuación de esta nueva modalidad de traducción que cobra una mayor atención en el mundo digital. Jiménez Crespo (2017: 25) propone, creemos que de forma muy acertada, la siguiente definición para solventar el dilema conceptual: «collaborative translation processes performed through dedicated web platforms that are initiated by companies or organizations and in which participants collaborate with motivations other than those strictly monetary». De otro lado, el término online collaborative translation queda definido por el mismo autor como "collaborative translation processes in the web initiated by self-organized online communities in which participants collaborate with motivations other than monetary» (Jiménez Crespo, 2017: 76).

En cuanto a las principales diferencias entre crowdsourcing translation y online collaborative translation, y siempre teniendo en cuenta las definiciones de los distintos trabajos mencionados, podemos afirmar que radican en el punto de inicio o de partida Así, en el caso de crowsourcing translation, son las propias empresas e instituciones las que inician el proceso de traducción y fijan su objetivo en la reducción de costes de producción (i.e. Facebook, Twitter, o Linkedln); por otro lado, online collaborative translation tiene su punto de partida en voluntarios que, motivados por distintas razones, tienen como principal objetivo hacer que otras comunidades de usuarios puedan acceder a contenido en su propia lengua. La relación que se establece en el caso de crowdsourcing translation parte de un eje vertical y jerárquico, mientras que online collaborative transaltion se caracteriza por un eje horizontal donde el proceso se establece entre pares iguales y se acerca a actividades como la conocida fansubbing (Díaz-Cintas y Muñoz Sánchez, 2006; O'Hagan y Mangiron, 2013) o bien el trabajo colaborativo que se realiza en determinados sitios web, como Wikipedia (McDonough Dolmaya, 2012).

De igual forma que ocurre en instituciones, organizaciones y comunidades en línea, la traducción como actividad social ha ganado popularidad dentro de la disciplina de los estudios de traducción, y son variados los campos de investigación que se abren a su paso (Cronin, 2010; Desjardins, 2011; Fernández Costales, 2012; García, 2010; Jiménez Crespo, 2015, 2017; O'Hagan, 2011; Perrino, 2009). Algunos estudiosos centran su atención en describir las implicaciones de esta nueva modalidad de traducción dentro de los distintos programas de formación de traductores (Desjardins 2011; McDonough Dolmaya, 2012, Sánchez Ramos, 2015). Hay trabajos, como los Baker (2009), que debaten el papel del voluntariado en traducción, y otros que se centran en cuestiones más concretas como los factores que llevan a un grupo de voluntarios a implicarse en esta modalidad de traducción (Fernández Costales, 2013; O'Brien y Schäler, 2010). Dejardins (2013) ofrece un estudio descriptivo sobre la experiencia docente realizada 
con un grupo de asignaturas y que aboga por la integración de las redes sociales, en concreto Facebook, como parte de la formación de traductores. Sus conclusiones señalan que la incorporación de este tipo de tecnología favorece el trabajo en equipo y colaborativo entre los alumnos y la creación de una 'comunidad' dentro del entorno de aprendizaje, muy en la línea de las teorías socioconstructivistas. Désilets (2007) se centra en la figura del traductor y en los principales desafíos que la traducción colaborativa en línea puede presentar. La actitud de los profesionales de la traducción ante los nuevos entornos colaborativos es estudiada en el trabajo de Gough (2011) como parte de un trabajo realizado en su formación de posgrado. Sus conclusiones apuntan a un desconocimiento generalizado de estos entornos entre los profesionales de la traducción, así como tareas de traducción colaborativas prácticamente inexistentes entre los participantes del experimento.

Con todo, la traducción colaborativa se presenta como una actividad innovadora, que surge como respuesta a las nuevas tendencias y necesidades en la producción y difusión de la información, y a las nuevas formas comunicativas dentro de una sociedad multilingüe cada vez más globalizada y conectada, lo que transforma el proceso de traducción en un proceso más abierto y colaborativo. En nuestra opinión, la introducción de estas nuevas modalidades en la clase de traducción sigue muy de cerca los pasos marcados por el Espacio Europeo de Educación Superior (EEES), donde se fijan como objetivos la adquisición de competencias, entendiendo estas como un conjunto de conocimientos, habilidades y actitudes, y eminentemente orientado al proceso de aprendizaje del estudiante. El docente adquiere un papel de facilitador del aprendizaje, convirtiéndose así también en una figura relevante en el proceso.

En lo referido al campo de la didáctica de la traducción, las teorías socioconstructivistas lideradas por Kiraly (2000) abogan por prácticas traductológicas reales en forma de proyectos, como puede ser el caso de las tareas realizadas bajo los enfoques situacionales (Kelly, 2011: 395). Su teoría socioconstructivista promueve prácticas centradas en el alumno, que desarrollen la responsabilidad, la independencia y la búsqueda de alternativas (Kiraly, 2000: 33), que ayuden al alumno a forjar su proceso de aprendizaje. Bajo esta teoría, la didáctica de la traducción adquiere su condición más dinámica y se dirige hacia la construcción de conocimiento. El socioconstructivismo otorga gran importancia al trabajo colaborativo, al aprendizaje activo y auténtico y a las tareas y proyectos reales. Se atisba de forma clara un componente de los llamados aprendizajes situacionales (Vienne, 1994; Gouadec, 1993) en la obra de Kiraly (2000), ya que los entornos de aprendizaje por los que se apuestan en las teoría 
socioconstructivistas se ofrecen en contextos colaborativos donde los alumnos puede trabajar en situaciones que se asemejan a la práctica traductológica profesional, lo que contribuye a una interacción con las tecnologías y el desempeño de papeles en el ejercicio de la traducción profesional como gestores de proyectos, documentalistas, terminólogos, traductores, correctores, etc. Parece lógico, pues, pensar que el enfoque socioconstructivista está íntimamente ligado a la filosofía de la era digital y la Web2.0, en donde los usuarios, además de subir contenidos y difundir la información a través de Internet, interactúan y 'colaboran' entre ellos (Desjardins, 2011: 179).

\section{Metodología}

El principal objetivo de la investigación realizada era incorporar nuevas prácticas traductológicas, como es la traducción colaborativa en línea, dentro del aula de traducción, y más concretamente dentro del sílabo de tecnología y traducción, además de conocer la percepción de los estudiantes, y futuros profesionales de la traducción, hacia estas prácticas para así poder incorporarlas con una mayor eficiencia en la didáctica de la traducción. Dicha investigación formó parte de la asignatura Herramientas informáticas aplicadas a la traducción, asignatura obligatoria de 6 créditos ECTS del Grado en Lenguas Modernas y Traducción (Universidad de Alcalá, Madrid), que se imparte en el segundo cuatrimestre del $3^{\text {er }}$ curso; y tuvo lugar durante cuatro sesiones con un total de 6 horas repartidas de la siguiente forma: 1) 1 sesión sobre aspectos teóricos y técnicos de la traducción colaborativa en línea (1 hora); 2 sesiones prácticas sobre traducción colaborativa (actividad grupal y de 2 horas de duración cada una); 3) 1 sesión final en forma de debate (1 hora). La investigación ocupó la última parte de la asignatura, con lo que los alumnos contaban ya con un bagaje en distintas herramientas informáticas aplicadas a la traducción, como herramientas de traducción asistida. Además, habían cursado las asignaturas de Terminología y Traducción especializada (científico-técnica), donde habían practicado con herramientas de extracción terminológica y gestores de concordancias.

La metodología empleada se asienta en los pilares del socioconstrucivismo descritos en párrafos anteriores (Kiraly, 2000), y donde los alumnos eran los responsables de su propio aprendizaje, además de organizar las actividades propuestas siguiendo muy de cerca la llamada investigación-acción.

Los sujetos que tomaron parte de esta investigación fueron 40 alumnos matriculados de la asignatura mencionada previamente, 32 de género femenino y 8 de género masculino, y que forma parte del plan de 
estudios del Grado en Lenguas Modernas y Traducción (Universidad de Alcalá).

El tipo de investigación empleado se denomina investigación-acción. Se trata de una metodología de corte cualitativo que se caracteriza por el papel activo que adquiere la figura del profesor como principal investigador y actor de cambio. Latorre (2003: 24) la define como "una indagación práctica realizada por el profesorado, de forma colaborativa, con la finalidad de mejorar su práctica educativa a través de ciclos de acción y reflexión". A su vez, la investigación-acción se distingue de otras investigaciones, principalmente en que:

-Requiere una acción como parte integrante del mismo proceso de investigación.

-El foco reside en los valores de la profesional y el profesional más que en las consideraciones metodológicas.

-Es una investigación sobre la persona, en el sentido de que los profesionales investigan sus propias acciones. (Latorre, 2003: 28)

La investigación-acción incluye además ciertos mecanismos metodológicos que sirven para destacarla y diferenciarla de otras investigaciones:

La investigación-acción se suele conceptuar como un "proyecto de acción" formado por "estrategias de acción", vinculadas a las necesidades del profesorado investigador y/o equipos de investigación. Es un proceso que se caracteriza por su carácter cíclico, que implica un "vaivén" —espiral dialéctica- entre la acción y la reflexión, de manera que ambos momentos quedan integrados y se complementan. El proceso es flexible e interactivo en todas las fases o pasos del ciclo. (Latorre, 2003: 32)

Este marco metodológico fundamentado en una «espiral de ciclos» fue establecido por Lewin (1946, en Latorre, 2003), quien describe el proceso investigador como una espiral de pasos o ciclos de acción reflexiva, que consiste en una serie de fases: planificación, implementación y evaluación de la acción. Siguiendo estas directrices, desglosaremos estas tres fases en un total de ocho pasos al igual que hacen Cohen y Manion (1990-289-291):

- Identificación de un problema y diagnóstico. El primer paso consiste en la identificación del problema por medio de los instrumentos apropiados y la formulación del diagnóstico. Para identificación y diagnóstico, en nuestro caso concreto, partimos de un test inicial realizado antes de la puesta en práctica de la primera sesión docente sobre traducción 
colaborativa en línea. Dicho test denota unas carencias notables en cuanto al conocimiento de aspectos relacionados con la traducción colaborativa en línea (plataformas colaborativas, archivos po, portable object, sistema gettext, etc.).

- Desarrollo de una propuesta de solución, y formulación de la hipótesis de acción o acción estratégica que se pretende demostrar. Nuestra propuesta de solución está relacionada con la puesta en marcha de un módulo enfocado a la traducción colaborativa en línea. La hipótesis de partida que se plantea es que un tratamiento pedagógico basado en la enseñanza explícita de la traducción colaborativa en línea aportará beneficios significativos a un grupo de alumnos de traducción en lo que a reflexión de nuevas prácticas traductológicas se refiere y concienciación de nuevas prácticas profesionales. La confirmación de la hipótesis supondría, asimismo, un primer paso para animar a otros docentes a comprobar dicha hipótesis en otros grupos y, de este modo, proceder a una generalización (gradual) de los resultados o, en su defecto, a la desestimación de los mismos.

- Planificación de los procedimientos de intervención/tratamiento pedagógico, en donde se especifican los distintos procedimientos que se van a realizar en la intervención o tratamiento pedagógico.

- Tratamiento pedagógico e intervención instructiva. En este paso se detalla el contexto (medio, lugar y temporalización) en el que se desarrolla el tratamiento, así como el diseño de apartados y actividades que forman parte del mismo.

- Recogida de datos mediante procedimientos - cuantitativos y cualitativos - de evaluación planificados. Además de un test inicial y otro final (procedimientos cuantitativos), se plantea un seguimiento de los alumnos a través de la introspección y (auto)valoración del proceso y producto traductor en forma de debate y grupos de discusión (procedimientos cualitativos).

- Análisis e interpretación de los resultados obtenidos, tanto de los cuantitativos como de los cualitativos.

- Confirmación o refutación de la hipótesis que se planteó ante el problema diagnosticado.

- Elaboración de conclusiones, a través de una reflexión sobre los anteriores pasos del proceso y proponiendo futuras investigaciones que se puedan inferir de los resultados obtenidos o del desarrollo mismo de la investigación actual. 
El tratamiento recibido consistió en realizar un trabajo grupal de tareas de traducción y revisión a través de distintas plataformas colaborativas. Así, la clase quedó dividida en 8 grupos de 5 alumnos. Cuatro grupos trabajaron con las llamadas crowdsourcing translation platforms y el resto de grupos con las llamadas collaborative translation platforms, siguiendo la clasificación propuesta por Jiménez Crespo (2017). Se trató principalmente de realizar traducciones y revisiones con las plataformas Trommons, ejemplo de crowdsourcing translation platform, y Pootle, ejemplo de collaborative translation platform. En el primer caso, los alumnos trabajaron en la traducción y revisión de textos cuya temática versaba sobre las necesidades de distintas organizaciones internacionales, y en el caso de Pootle, los grupos realizaron tareas de traducción y revisión de software de código abierto y trabajaron con archivos .po. Tras la realización de la actividad, y como principales instrumentos de recogida de datos, se distribuyó un cuestionario final. Igualmente, se dedicó una hora de clase a debatir la tarea realizada y reflexionar sobre las ventajas, desventajas y principales desafíos de esta práctica traductológica.

\section{RESULTADOS}

El test inicial estaba formado por un total de cinco preguntas. Las tres primeras estaban destinadas, en una escala Likert del 1 al 7 , a que el alumno se autoevaluara como 1) usuario de Internet; 2) usuarios de herramientas informáticas en general 3) satisfacción con los conocimientos informáticos adquiridos durante sus estudios de grado actuales. Como puede observarse de Imágenes 1.1 y 1.2 , el alumnado parecía mostrar una actitud confiada en el manejo de herramientas informáticas y los conocimientos adquiridos hasta ahora durante el Grado en Lenguas Modernas y Traducción. Hay que resaltar que la asignatura de Herramientas informáticas aplicadas a la traducción se cursa en el segundo cuatrimestre del $3^{\text {er }}$ curso, y es de carácter obligatorio. En este sentido es importante señalar que, previo a cursar esta asignatura, el alumnado ha cursado las asignaturas de Terminología y Traducción especializada (científico-técnica), donde han trabajado con SDL Multiterm, como herramienta de gestión terminológica. 


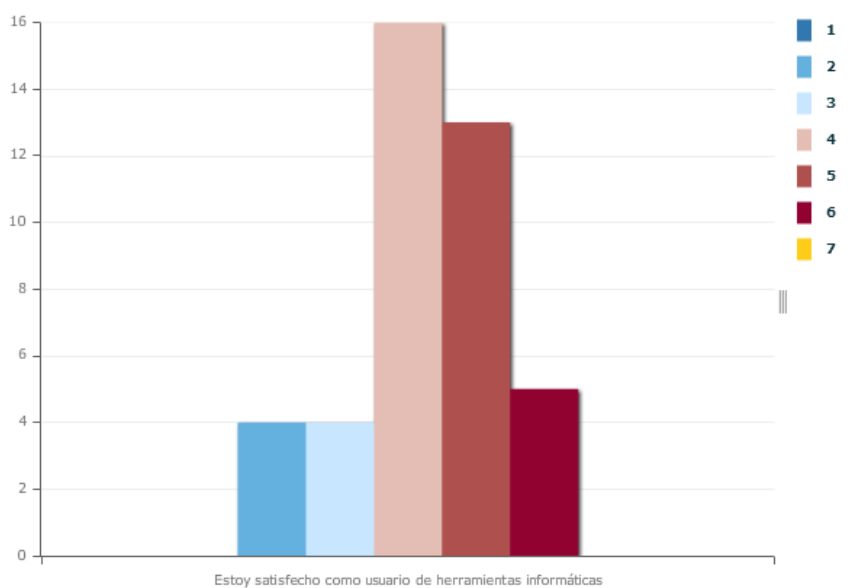

Imagen 1.1. «Satisfacción del alumnado como usuario de herramientas informáticas»

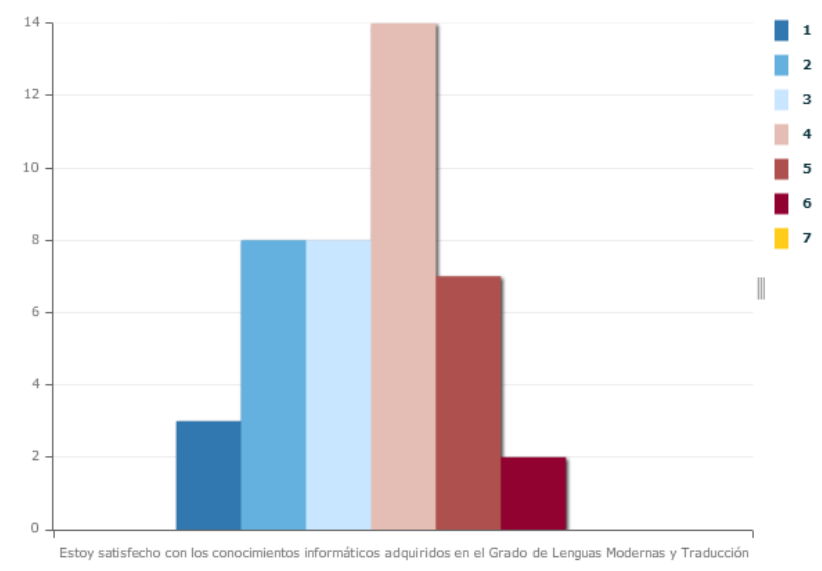

Imagen 1.2. «Satisfacción del alumno conocimientos informáticos adquiridos en el Grado de Lenguas Modernas y Traducción»

Las últimas dos preguntas estaban destinadas a indagar sobre el conocimiento que los sujetos tenían de la traducción colaborativa en línea (Pregunta 4) y las plataformas las plataformas o herramientas informáticas colaborativas (Pregunta 5). En el primer caso, 10 alumnos contestaron de 
forma afirmativa, si bien no se les pedía una definición de la misma al considerarse innecesario, y 30 indicaron que desconocían la traducción colaborativa en línea. En cuanto a la pregunta 5, tan solo 10 alumnos contestaron de forma correcta a la misma e indicaron Facebook como ejemplo, el resto de los sujetos proporcionaron respuestas que no se ajustaban a ninguna plataforma o herramienta o bien dejaron la pregunta sin contestar.

En lo referido al debate, la puesta en marcha de la actividad sin duda ha incidido de forma positiva tanto en la docencia de la asignatura como en la metodología empleada y la consecución de objetivos. Uno de los principales objetivos de la actividad, y en concreto el debate, era que los sujetos reflexionaran sobre los distintos aspectos y lo que conlleva la traducción colaborativa en línea e iniciar, de este modo, un debate sobre nuevas prácticas traductoras y sus implicaciones en el mercado profesional. Se perseguía, por tanto, una reflexión crítica hace nuevas modalidades (o posibilidades) de traducción reales que los alumnos, en un futuro muy cercano, podían llegar a enfrentarse. La recogida de datos en la sesión del debate se realizó mediante la toma de notas y la observación por parte del profesor. De dichos datos se desprende que los sujetos eran conscientes de las ventajas y desventajas de la traducción colaborativa en línea, además de iniciarse un debate sobre temas como cuestiones éticas, las relacionadas con la calidad del producto final en las tareas de traducción colaborativa o el componente social de la traducción colaborativa en lo referido a lenguas minoritarias. La sesión dedicada al debate puso sobre la mesa también la influencia de la traducción colaborativa en el mundo profesional de la traducción. Como aspectos positivos, los sujetos señalaron las tareas de compromiso y responsabilidad social. Los alumnos expusieron distintos puntos de vista, expresaron variedad de opiniones, favoreciendo de este modo al desarrollo de un pensamiento crítico y reflexión sobre nuevas modalidades de traducción. La puesta en marcha de este tipo de actividades contribuyó a la mejora del trabajo grupal, así como una mejor interacción entre el proceso de comunicación docente-alumno o el fomento de una actitud positiva hacia las tareas en grupo. Algunos de los comentarios recogidos fueron: «Desconocía que se podía ayudar de esta forma a través de Internet, pero alguien revisará mi traducción» (sujeto 1); «Creo que se necesita la asignatura para poder hacer esta actividad con el programa [Pootle] (sujeto 2 ); "deberían pagar y no tener que traducir gratis» (sujeto 3); "este tipo de traducción quizás sea más beneficioso para las lenguas minoritarias» (sujeto 4); «me ha gustado trabajar con mis compañeros en un proyecto real» (sujeto $5)$. 
Por último, y con el fin de evaluar nuestra propuesta y la metodología empleada, se diseñó un test final formado por cinco preguntas. Los 40 alumnos cumplimentaron el cuestionario. Como puede desprenderse de la primera pregunta (Ahora que conoces la traducción colaborativa en línea, ¿consideras que es una práctica traductora a tener en cuenta en un futuro?), la actitud de los sujetos hacia la traducción colaborativa en línea demuestra que es una actividad a tener en cuenta, lo que coincide con otros estudios (Gough, 2011):

\begin{tabular}{|l|r|}
\hline Sí, mucho & $66 \%$ \\
\hline Sí, algo & $14 \%$ \\
\hline Sí, poco & $17 \%$ \\
\hline No, nada & $3 \%$ \\
\hline Total & $100 \%$ \\
\hline
\end{tabular}

Tabla 1 : Pregunta 1 (Ahora que conoces la traducción colaborativa en línea, ¿consideras que una práctica traductora a tener en cuenta en un futuro?)

La segunda pregunta venía a corroborar lo indicado en la sesión de debate. $Y$ es que, aunque el alumnado era consciente de estas prácticas, también era reticente a las mismas, tal y como también señala el estudio de Gough (2011). Ello es debido, según quedó señalado en la sesión de debate, a cuestiones éticas relacionadas con la profesionalización de la traducción, por ejemplo. Aquellos que indicaron que tenían intención de formar parte de alguna actividad de traducción colaborativa señalaban que solo lo harían de manera esporádica y, algunos de ellos, como ejercicio de la práctica de la traducción.

\begin{tabular}{|l|l|}
\hline Sí & $25 \%$ \\
\hline No estoy seguro & $45 \%$ \\
\hline No & $30 \%$ \\
\hline Total & $100 \%$ \\
\hline
\end{tabular}

Tabla 2: Pregunta 2 (Tras la actividad sobre traducción colaborativa en línea, ¿crees que formarás parte de iniciativas o proyectos de esta índole? 
La tercera pregunta puntualizaba aspectos debatidos en la sesión final de la actividad. Si bien la traducción colaborativa en línea puede generar cierta reticencia, se atisba como necesaria en un mundo cada vez más globalizado y multilingüe, donde el acceso a la información debe llegar a todas las comunidades lingüísticas.

\begin{tabular}{|l|r|}
\hline Muy útil & $\mathbf{9 0 , 9 0 \%}$ \\
\hline Más útil & $6,06 \%$ \\
\hline Igual & $3,03 \%$ \\
\hline Menos útil & $0 \%$ \\
\hline Total & $100 \%$ \\
\hline
\end{tabular}

Tabla 3 : Pregunta 3 (¿Consideras que la traducción colaborativa en línea es necesaria y útil en el caso de las lenguas minoritarias?)

La pregunta 4 era una pregunta abierta en la que se pedía a los sujetos indicar la que para ellos era la principal desventaja de la traducción colaborativa en línea. Entre las distintas respuestas, los sujetos subrayaron la falta de control de calidad y los problemas derivados de la remuneración de las traducciones y la práctica profesional, datos que concordaban con las conclusiones extraídas de la sesión del debate.

Por último, la pregunta 5 estaba destinada a conocer si este tipo de actividades debía incorporarse a los contenidos de la asignatura de Herramientas informáticas aplicadas a la traducción. La gran mayoría del alumnado estaba a favor de incluirlas como parte de la práctica docente, al igual que el estudio de McDonough Dolmaya (2012) o Desjardins (2011). Sí hay que matizar que, obviamente, la incorporación de estas actividades dependerá de ciertos factores, como el propio plan de estudios o las distintas asignaturas enfocadas en traducción y tecnología. En nuestro caso concreto, la asignatura es la única con un contenido más técnico, por lo que se ha considerado la incorporación de dichas prácticas. 


\begin{tabular}{|l|r|}
\hline Mucho & $\mathbf{9 0 , 9 0 \%}$ \\
\hline Bastante & $6,06 \%$ \\
\hline Poco & $3,03 \%$ \\
\hline Nada & $0 \%$ \\
\hline Total & $100 \%$ \\
\hline
\end{tabular}

Tabla 4 : Pregunta 5 (¿Crees necesaria la formación en nuevas prácticas traductoras, como es el caso de la traducción colaborativa en línea, en la formación del Grado en Lenguas Modernas y Traducción?

\section{CONCLUSIONES}

La colaboración en distintos ámbitos, en donde se incluye la esfera comunicativa, es prácticamente un hecho inherente a la condición humana. Y la traducción no es una excepción. La propia traducción como proceso de intercambio de información es un ejemplo de colaboración lingüística. Los docentes implicados en la didáctica de la tecnología y la traducción deben ser conscientes de las nuevas formas de traducción que han comenzado a ser centro de debate. El presente trabajo ha querido poner de manifiesto la importancia que nuevas modalidades de traducción están teniendo en el mundo profesional de la traducción, como es la traducción colaborativa en línea. Así, bajo un enfoque socioconstructivista y una metodología basada en la llamada investigación-acción, se han presentado los datos cuantitativos y cualitativos de la investigación realizada en el aula de la asignatura Herramientas informáticas aplicadas a la traducción, como parte del Grado en Lenguas Modernas y Traducción (Universidad de Alcalá). Ello nos ha permitido obtener información sobre el desconocimiento inicial que los alumnos tenían sobre prácticas de traducción colaborativa (test inicial) y el cambio de actitud que, tras el tratamiento pedagógico realizado, los sujetos experimentaron (test final).

La experiencia docente ha hecho del aula un espacio abierto en el que trabajar de forma colaborativa con el resto de los compañeros. Dicha experiencia ha permitido crear una atmósfera de aprendizaje inigualable para el intercambio de opiniones y el desarrollo de un espíritu crítico ante nuevas formas de traducción. A nuestro entender, y como docentes implicados a favor de la integración de la tecnología y traducción en la formación de nuestros estudiantes, se hace imprescindible exponer a los estudiantes no solo a los nuevos avances del mundo de la traducción profesional sino contribuir a la elaboración de un sílabo que esté acorde con dichos avances. Aunque cuestiones como la calidad de la traducción en estos entornos o el 
hecho de que no sea una actividad remunerada pueda contribuir a dejar de lado estas tareas, no podemos negar se trata de una actividad que se está abriendo camino y que no es algo pasajero. Consideramos que este trabajo, para nada conclusivo, puede ser el inicio de investigaciones futuras y que contribuya a abrir el debate ya iniciado sobre la traducción colaborativa y su repercusión en los estudios de traducción.

\section{REFERENCIAS BIBLIOGRÁFICAS}

Baker, M. (2009). Forget neutrality. The Linguist, 48:3, pp. 24-26.

Cordingley, A. y Manning, C. F. (2016). Collaborative translation: From the Renaissance to the digital age. Londres: Bloomsbury Publishing.

Cohen, L. y Manion, L. 1990. Métodos de investigación educativa. Madrid: La Muralla.

Cronin, M. (2010). The translation crowd. Revista Tradumàtica, 8, pp. 1-7.

Declerq, C. (2014). Crowd, cloud and automation in the translation education community. Cultus, 7, 37-56.

DePalma, D. y Kelly, N. (2008). Translation of, for, and by the people: how user-translated content projects work in real life. Common Sense Advisory Reports. Recuperado de: https://www.commonsenseadvisory.com/Portals/_default/Knowledgeb ase/Articlelmages/081218_R_community_trans_Preview.pdf

Désilets, A. (2007). Translation Wikified: How will massive online collaboration impact the world of translation?" Translating and the computer, 29. Recuperado de: http://www.mt-archive.info/Aslib-2007-Desilets.pdf

Desjardins, R. (2011). Facebook me!: Initial insights in favour of using social networking as a tool for translator training". Linguistica antverpiensia, 10, pp. 175-192.

Díaz-Cintas, J. y Muñoz Sánchez, P. (2006). Fansubs: audiovisual translation in an amateur environment. The Journal of specialised translation 6, pp. 37-52.

Drugan, J. (2013). Quality in professional translation: assessment and improvement. Londres. Bloomsbury Publishing.

Fernández Costales, F. (2012). Collaborative translation revisited: exploring the rationale and the motivation for volunteer translation. Forum, 10:1, pp. 115-142. 
Fernández Costales, Fernando. (2013). Crowdsourcing and collaborative translation: mass phenomena or silent threat to translation studies?" Hermeneus, 15, pp. 85-110.

Folaron, D. (2006). A discipline coming of age in the digital age. In K. Dunne (Ed.), Perspectives on localization (pp. 195-219). Amsterdam/Philadelphia: John Benjamins.

García, I. (2010). The proper place of professionals (and non-professionals and machines) in web translation. Revista Tradumática, 8.

Gaspari, F. (2015). Online translation. En Sin-wai (ed.). Routledge encyclopedia of translation technology (pp. 578-593). Nueva YorkLondres: Routledge.

Gouadec, D. (2007). Translation as a profession. Ámsterdan/Filadelfia: John Benjamins Publishing.

Gough, J. (2011). An empirical study of professional translators' attitudes, use and awareness of Web 2.0 technologies, and implications for the adoption of emerging technologies and trends. Linguistica antverpiensia, 10, pp. 195-217.

Howe, J (2006). Crowdsourcing: A definition. Wired Blog Network: Crowdsourcing, $14 . \quad$ Recuperado de: http://crowdsourcing.typepad.com/cs/2006/06/crowdsourcing_a.html

Jiménez-Crespo, M. A. (2015). The Internet in translation education: two decades later. TIIS: Translation and interpreting studies, 10, pp. 33-57.

Jiménez- Crespo, M. A. (2017). Crowdsourcing and online collaborative translations. Ámsterda/Filadelfia: John Benjamins Publishing.

Kelly, D. (2002). "Un modelo de competencia traductora: bases para el diseño curricular". Puentes, 1, pp. 9-20.

Kiraly, D. (2000). A social constructivist approach to translator education: empowerment from theory to practice. Manchester: St. Jerome.

Latorre, A. (2003). La investigación-acción. Conocer y cambiar la práctica educativa. Barcelona: Graó.

McDonough Dolmaya, J. (2012). Analyzing the crowdsourcing model and its impact on public perceptions of translation. The Translator, 18:2, pp. 167-191. 
O’Brien, S. (2011). Collaborative translation. En C. Millán y F. Bartrina (eds.). Routledge handbook of translation studies (pp. 17-20). Nueva YorkLondres: Routledge.

O'Brien, S. y Schäler, R. (2010). Next generation Translation and localisation: Users are taking care. Translating and the Computer. Recuperado de: http://doras.dcu.ie/16695/1/Paper_6.pdf

O'Hagan, M. (2011). Introduction: Community translation: Translation as a social activity and its possible consequences in the advent of Web 2.0 and beyond. Linguistica antverpiensia, 10, pp.1-10.

O'Hagan, M. (2013). The impact of new technologies on translation studies. A technological turn? En C. Millán \& F. Bartrina (eds.). The Routledege handbook of translation studies (pp. 503-518). Londres: Routledge.

O'Hagan, M. \& Mangiron, C. (2013). Videogame localization. Ámsterdan/Filadelfia: John Benjamins.

O'Reilly, T. (2005). What is Web 2.0? Design patterns and business models for the next generation of software. Recuperado de: http://www.oreilly.com/pub/a/web2/archive/what-is-web20.html?page $=1$

Perrino, S. (2009). User-generated translation: the future of translation in a Web 2.0 environment. The Journal of specialised translation, 12, pp. 55-78.

Pym, A. (2011). Translation research terms: a tentative glossary for moments of perplexity and dispute. En A. Pym (ed.). Translation research projects 3. Tarragona: Intercultural Studies Group, pp. 75-110.

Ray, R., y Kelly, N. (2011). Crowdsourced translation: Best practices for implementation. Lowell, MA: Common Sense Advisory.

Rico, C. (2017). The ePortfolio: constructing learning in translation technology. The Interpreter and Translator Trainer, 11, 11, pp: 79-95.

Schäler, R. (2011). Localization. En M. Baker \& G. Saldanha. (Eds.). Encyclopedia of Translation Studies, (pp. 157-161). Nueva YorkLondres: Routledge.

Sánchez Ramos, M. M. (2015). Exploring social translation and ethics in the classroom: some implications for translator training. En S. Izwaini (ed.). Papers in Translation Studies (pp. 96-113). Cambridge: Cambridge Scholars Publishers. 
Tawileh, A. (2010). Managing social translation: online tools for translators' ccmmunities. Translating and the computer, 32. Recuperado de: http://mt-archive.info/Aslib-2010-Tawileh.pdf

Vienne, J. (1994). Towards a pedagogy of translation in situation. Perspectives, 2:1, pp. 51-59. 